\title{
Static output feedback control for continuous-time TS descriptor models: decoupling the Lyapunov function
}

\author{
Víctor Estrada-Manzo, Thierry Marie Guerra \\ LAMIH UMR CNRS 8201 \\ University of Valenciennes and Hainaut-Cambresis \\ Le Mont Houy, 59300, Valenciennes, France \\ \{victor.estradamanzo,guerra\}@univ-valenciennes.fr
}

\author{
Zsófia Lendek \\ Department of Automation \\ Technical University of Cluj-Napoca \\ Memorandumului 28, 400114, Cluj-Napoca, Romania \\ zsofia.lendek@aut.utcluj.ro
}

\begin{abstract}
The paper deals with static output feedback controller design for continuous-time Takagi-Sugeno descriptor models. Via the well-known Finsler's Lemma and the descriptorredundancy approach a set of linear matrix inequalities are derived to solve this design problem. A numerical example shows the effectiveness of the proposed approaches.
\end{abstract}

Keywords - Takagi-Sugeno descriptor models; output feedback control; linear matrix inequalities; Finsler's Lemma.

\section{INTRODUCTION}

The use of Takagi-Sugeno (TS) models [1] have been widely employed. Thanks to their convex structure, the Lyapunov theory has been used for the analysis TS models [2]; analysis and design conditions have yielded linear matrix inequality (LMI) constraints, which are solved via convex optimization techniques [3]. Since the appearance of the sector nonlinearity methodology [4], researchers have paid more attention to TS modelling, due to their capability to exactly represent a nonlinear system [5], [6]. Nevertheless, the sector nonlinearity presents an important disadvantage: when the original nonlinear model contains a high number of nonconstant terms, the number of rules rapidly increases, thus turning the problem numerically intractable.

Expressing analysis and designing conditions as LMIs is one of the main goals of the TS framework. Several works concerning controller/observer design of continuous-time TS models [7]-[17] have been developed. For the discrete-time, the use of non-quadratic Lyapunov functions has led to important results [18]-[23].

Based on nonlinear descriptor models [24] — which naturally appear in mechanical systems [25] — TS descriptors have been introduced in [26]. Using a TS descriptor representation generally reduces the high number of rules while the TS model remains closer to the structure of the original nonlinear model [27], [28].

One of the most challenging problems in automatic control is the design of output feedback control. A survey on the design of static output feedback controller (SOFC) for linear systems has been presented in [29]; later on, an iterative LMI (ILMI) procedure has been presented in [30], further sufficient LMI conditions have been developed in [31]. In [32]-[34], the results for linear systems have been extended for standard TS models. More recently, via the descriptor-redundancy approach and Finsler's Lemma, LMI conditions have been achieved. Both in the continuous-time case [34], [35] and in the discretetime case [36]. In [37], SOFC conditions for the discrete-time TS descriptors have been developed.

Despite these works, there are no results concerning the SOFC design for TS descriptor models in continuous time. This paper intends to fill this gap. To this end, the SOFC design is done via the well-known Finsler's Lemma, since it avoids using the classical closed-loop system for the design, thus breaking the link between the Lyapunov function and the controller. Moreover, taking advantage of this approach, slack variables can be directly added in order to reduce conservativeness. The selection of these slack matrices is inspired by [36], [37], such that they provide the maximum flexibility to the LMI constraints (the maximum no. of decision variables but keeping the same number of convex sums).

The rest of the paper is organized as follows: Section II presents the TS descriptor model, notation, and the problem statement; Section III states the main results and some remarks on the current research; Section IV illustrates the provided approaches; Section $\mathrm{V}$ concludes the paper.

\section{PRELIMINARIES}

\section{A. Notation}

Consider a set of membership functions (MFs) $h_{i}(\bullet)$, $i \in\{1,2, \ldots, r\}$, which hold the convex-sum property, i.e., $0 \leq h_{i}(\bullet) \leq 1, i \in\{1,2, \ldots, r\}$, and $\sum_{i=1}^{r} h_{i}(\bullet)=1$. Throughout this work a shorthand notation will be used to represent convex-sums of matrix expressions:

$$
\begin{gathered}
\Upsilon_{h}=\sum_{i=1}^{r} h_{i}(\bullet) \Upsilon_{i}, \Upsilon_{h}^{-1}=\left(\sum_{i=1}^{r} h_{i}(\bullet) \Upsilon_{i}\right)^{-1}, \\
\Upsilon_{h h}=\sum_{i=1}^{r} \sum_{j=1}^{r} h_{i}(\bullet) h_{j}(\bullet) \Upsilon_{i j},
\end{gathered}
$$

where $\Upsilon_{i}, \Upsilon_{i j}, i, j \in\{1,2 \ldots, r\}$ are matrices of appropriate dimensions. Subscripts may change according to the MFs. In addition, an asterisk $(*)$ will be used in matrix expressions to 
denote the transpose of the symmetric element. For in-line expressions it will denote the transpose of the terms on its lefthand-side, for example:

$$
\left[\begin{array}{cc}
A & B^{T} \\
B & C
\end{array}\right]=\left[\begin{array}{cc}
A & (*) \\
B & C
\end{array}\right], \quad A+B+A^{T}+B^{T}=A+B+(*) .
$$

\section{B. TS descriptor model}

Consider the following continuous-time nonlinear descriptor model:

$$
\begin{aligned}
E(x(t)) \dot{x}(t) & =A(x(t)) x(t)+B(x(t)) u(t) \\
y(t) & =C(x(t)) x(t),
\end{aligned}
$$

where $x(t) \in \mathbb{R}^{n}$ is the state vector, $u(t) \in \mathbb{R}^{m}$ is the control input, and $y(t) \in \mathbb{R}^{o}$ is the output vector. Matrices $A(x(t))$, $B(x(t)), C(x(t))$, and $E(x(t))$ are assumed to be bounded and smooth in the compact set $\Omega_{x}$. This work studies the case when $E(x(t))$ is a nonsingular matrix in $\Omega_{x}$. In the sequel, arguments will be omitted when their meaning can be inferred from the context.

Employing the sector nonlinearity methodology [4], the $p$ non-constant terms in $(A(x), B(x), C(x))$ are grouped in MFs $h_{i}(\bullet), i \in\left\{1,2, \ldots, 2^{p}\right\}$; similarly the $p_{e}$ nonlinearities on the left-hand side of (1) produce $v_{k}(\bullet), k \in\left\{1,2, \ldots, 2^{p_{e}}\right\}$. These MFs satisfy the convex sum property in $\Omega_{x}$ :

$$
\begin{aligned}
& 0 \leq h_{i}(\bullet) \leq 1, \quad \sum_{i=1}^{r} h_{i}(\bullet)=1, \\
& 0 \leq v_{k}(\bullet) \leq 1, \quad \sum_{k=1}^{r_{e}} v_{k}(\bullet)=1 .
\end{aligned}
$$

Using the mentioned methodology, an exact representation in $\Omega_{x}$ of the nonlinear model (1) is given by the following TS model in the descriptor form [26]:

$$
\begin{aligned}
\sum_{k=1}^{r_{e}} v_{k}(z) E_{k} \dot{x} & =\sum_{i=1}^{r} h_{i}(z)\left(A_{i} x+B_{i} u\right) \\
y & =\sum_{i=1}^{r} h_{i}(z) C_{i} x
\end{aligned}
$$

where the set of matrices $\left(A_{i}, B_{i}, C_{i}\right), i \in\{1,2, \ldots, r\}$ represent the linear local models in right-hand-side of the TS descriptor model, while $E_{k}, k \in\left\{1,2, \ldots, r_{e}\right\}$ denote the linear local models in the left-hand-side of (2). The premise variables are grouped in the vector $z(t)$, which is considered known. Using the shorthand notation, the TS descriptor model is written as $E_{v} \dot{x}=A_{h} x+B_{h} u, \quad y=C_{h} x$. Note that, if $E_{v}=I_{n}$, the standard TS model is recovered:

$$
\dot{x}=A_{h} x+B_{h} u, \quad y=C_{h} x .
$$

\section{Previous results}

For standard TS representations (see (3)), an ILMI approach and sufficient LMI conditions have developed in [32], [33]. In particular, the authors in [33] design a parallel distributed compensation (PDC) control law of the form:

$$
u=K_{h} y=K_{h} C_{h} x .
$$

Their analysis relies on the closed-loop system

$$
\dot{x}=\left(A_{h}+B_{h} K_{h} C_{h}\right) x .
$$

Stabilization conditions are given in terms of LMIs together with equalities:

$$
\begin{gathered}
A_{h} P+B_{h} N_{h} C_{h}+(*)<0, \quad M C_{h}-C_{h} P=0, \\
N_{h}=K_{h} M, \quad P=P^{T}>0 .
\end{gathered}
$$

Conditions (6) are similar as those in [31]. In [34], via the descriptor-redundancy approach, LMI conditions for stabilization of a TS model (3) via a non-PDC control law $u=K_{h}\left(P_{1 h}\right)^{-1} y$ have been obtained.

\section{Problem statement}

Descriptor models appear when dealing with mechanical systems; since they are more general than the standard ones, a TS descriptor representation comes at hand. The aim is to control the TS descriptor model (2) via SOFC of the following form:

$$
u=H_{h h v}^{-1} K_{h v} y=H_{h h v}^{-1} K_{h v} C_{h} x,
$$

where $H_{h h v} \in \mathbb{R}^{m \times m}$ and $K_{h v} \in \mathbb{R}^{m \times o}$ are nonlinear gains: they include nonlinearities from both sides of the TS descriptor model. Note that the control law (7) includes (4).

Using Finsler's Lemma, it is possible to decouple the Lyapunov function and the controller; this procedure allows achieving LMI conditions; as a result, it avoids the equality conditions in [33] and the ILMI conditions in [32].

Lemma 1 [38]: (Finsler's Lemma) Let $\mathcal{X} \in \mathbb{R}^{n}$, $\mathcal{Q}=\mathcal{Q}^{T} \in \mathbb{R}^{n \times n}$, and $\mathcal{R} \in \mathbb{R}^{m \times n}$ such that $\operatorname{rank}(\mathcal{R})<n$; the following expressions are equivalent:

$$
\begin{aligned}
& \text { a) } \mathcal{X}^{T} \mathcal{Q} \mathcal{X}<0, \quad \forall \mathcal{X} \in\left\{\mathcal{X} \in \mathbb{R}^{n}: \mathcal{X} \neq 0, \mathcal{R} \mathcal{X}=0\right\} \\
& \text { b) } \exists \mathcal{M} \in \mathbb{R}^{n \times m}: \mathcal{M} \mathcal{R}+\mathcal{R}^{T} \mathcal{M}^{T}+\mathcal{Q}<0 .
\end{aligned}
$$

In order to get LMI constraints, MFs should be dropped off from inequalities; to this end sum relaxations are available. Results in this paper consider the following sum relaxation scheme.

Lemma 2 [39]: Let $\Upsilon_{i j}^{k}, i, j \in\{1,2, \ldots, r\}, k \in\left\{1,2, \ldots, r_{e}\right\}$, be matrices of appropriate dimensions. Then $\Upsilon_{h h}^{v}=\sum_{i=1}^{r} \sum_{j=1}^{r} \sum_{k=1}^{r_{e}} h_{i}(z) h_{j}(z) v_{k}(z) \Upsilon_{i j}^{k}<0$ holds if

$$
\Upsilon_{i i}^{k}<0, \forall i, k ; \quad \frac{2}{r-1} \Upsilon_{i i}^{k}+\Upsilon_{i j}^{k}+\Upsilon_{i j}^{k}<0, i \neq j
$$




\section{MAIN Results}

In order to use Finsler's Lemma [38], the TS descriptor model together with the control law are written as the following equality constraint:

$$
\left[\begin{array}{ccc}
A_{h} & -E_{v} & B_{h} \\
H_{h h v}^{-1} K_{h v} C_{h} & 0 & -I
\end{array}\right]\left[\begin{array}{c}
x \\
\dot{x} \\
u
\end{array}\right]=0 .
$$

This rewriting avoids the explicit appearance of the classical closed-loop: $E_{v} \dot{x}=\left(A_{h}+B_{h} H_{h h v}^{-1} K_{h v} C_{h}\right) x$, and it also decouples the nonlinear matrix $E_{v}$.

The Lyapunov function under consideration is

$$
V(x)=x^{T} P x, \quad P=P^{T}>0 .
$$

Then, the time-derivative of (10) gives

$$
\dot{V}(x)=\dot{x}^{T} P x+x^{T} P \dot{x}<0 .
$$

Incorporating $u^{T} 0 u$ in the previous inequality, it gives $\dot{V}(x)=\dot{x}^{T} P x+x^{T} P \dot{x}+u^{T} 0 u<0$, which can be grouped as:

$$
\dot{V}(x)=\left[\begin{array}{c}
x \\
\dot{x} \\
u
\end{array}\right]^{T}\left[\begin{array}{lll}
0 & P & 0 \\
P & 0 & 0 \\
0 & 0 & 0
\end{array}\right]\left[\begin{array}{l}
x \\
\dot{x} \\
u
\end{array}\right]<0 .
$$

Via Lemma 1 (Finsler's Lemma), the inequality (12) under the equality constraint (9) gives:

$$
\mathcal{M}_{(\cdot)}\left[\begin{array}{ccc}
A_{h} & -E_{v} & B_{h} \\
H_{h h v}^{-1} K_{h v} C_{h} & 0 & -I
\end{array}\right]+(*)+\left[\begin{array}{ccc}
0 & P & 0 \\
P & 0 & 0 \\
0 & 0 & 0
\end{array}\right]<0
$$

with $\mathcal{M}_{(\cdot)} \in \mathbb{R}^{(2 n+m) \times(n+m)}$ being a free matrix:

$$
\mathcal{M}_{(\cdot)}=\left[\begin{array}{cc}
M_{1} & M_{2} \\
M_{3} & M_{4} \\
M_{5} & M_{6}
\end{array}\right] \text {. }
$$

Expression (13) yields

$$
\left[\begin{array}{ccc}
\Gamma^{(1,1)} & (*) & (*) \\
\Gamma^{(2,1)} & -M_{3} E_{v}-E_{v}^{T} M_{3}^{T} & (*) \\
\Gamma^{(3,1)} & -M_{5} E_{v}+\left(M_{3} B_{h}-M_{4}\right)^{T} & \Gamma^{(3,3)}
\end{array}\right]<0,
$$

with

$$
\begin{aligned}
& \Gamma^{(1,1)}=M_{1} A_{h}+M_{2} H_{h h}^{-1} K_{h v} C_{h}+(*), \\
& \Gamma^{(2,1)}=M_{3} A_{h}+M_{4} H_{h h v}^{-1} K_{h v} C_{h}+\left(P-M_{1} E_{v}\right)^{T}, \\
& \Gamma^{(3,1)}=M_{5} A_{h}+M_{6} H_{h h v}^{-1} K_{h v} C_{h}+\left(M_{1} B_{h}-M_{2}\right)^{T}, \\
& \Gamma^{(3,3)}=M_{5} B_{h}-M_{6}+(*) .
\end{aligned}
$$

Remark 1: The slack matrices in $M_{(\cdot)}$ can be chosen. Several options are available. The idea is to obtain an LMI problem; following the idea from [36], we propose $M_{2}=M_{4}=\eta H_{h h v}$ and $M_{6}=H_{h h v}$ where $\eta \in \mathbb{R}^{n \times m}$ is a constant matrix to be fixed in advance.

Theorem 1: The T-S descriptor model (2) under the control law (7) is asymptotically stable if there exist matrices $P=P^{T}>0, M_{1 j}, M_{3 j}, M_{5 j}, H_{i j k}, K_{j k}, i, j \in\{1,2, \ldots, r\}$, $k \in\left\{1,2, \ldots, r_{e}\right\}$ such that (8) with

$$
\Upsilon_{i j}^{k}=\left[\begin{array}{ccc}
\Gamma^{(1,1)} & (*) & (*) \\
\Gamma^{(2,1)} & -M_{3 j} E_{k}-E_{k}^{T} M_{3 j}^{T} & (*) \\
\Gamma^{(3,1)} & -M_{5 j} E_{k}+\left(M_{3 j} B_{i}-\eta H_{i j k}\right)^{T} & \Gamma^{(3,3)}
\end{array}\right],
$$

where

$$
\begin{aligned}
& \Gamma^{(1,1)}=M_{1 j} A_{i}+\eta K_{j k} C_{i}+(*), \\
& \Gamma^{(2,1)}=M_{3 j} A_{i}+\eta K_{j k} C_{i}+\left(P-M_{1 j} E_{k}\right)^{T}, \\
& \Gamma^{(3,1)}=M_{5 j} A_{i}+K_{j k} C_{i}+\left(M_{1 j} B_{i}-\eta H_{i j k}\right)^{T}, \\
& \Gamma^{(3,3)}=M_{5 j} B_{i}-H_{i j k}+(*) .
\end{aligned}
$$

Proof: Inequality (14) has been obtained via Lemma 1. Taking into account Remark 1 and using convex-sums, the slack matrices in $M_{(\cdot)}$ are chosen as follows:

$$
\begin{gathered}
M_{1 h}, M_{3 h} \in \mathbb{R}^{n \times n}, \quad M_{5 h} \in \mathbb{R}^{m \times n}, \\
M_{2 h h v}=M_{4 h h v}=\eta H_{h h v}, \quad M_{6 h h v}=H_{h h v} .
\end{gathered}
$$

With this selection (14) yields

$$
\Upsilon_{h h}^{v}:=\left[\begin{array}{ccc}
\Gamma^{(1,1)} & (*) & (*) \\
\Gamma^{(2,1)} & -M_{3 h} E_{v}-E_{v}^{T} M_{3 h}^{T} & (*) \\
\Gamma^{(3,1)} & -M_{5 h} E_{v}+\left(M_{3 h} B_{h}-\eta H_{h h v}\right)^{T} & \Gamma^{(3,3)}
\end{array}\right]<0
$$

where

$$
\begin{aligned}
& \Gamma^{(1,1)}=M_{1 h} A_{h}+\eta K_{h v} C_{h}+(*), \\
& \Gamma^{(2,1)}=M_{3 h} A_{h}+\eta K_{h v} C_{h}+\left(P-M_{1 h} E_{v}\right)^{T}, \\
& \Gamma^{(3,1)}=M_{5 h} A_{h}+K_{h v} C_{h}+\left(M_{1 h} B_{h}-\eta H_{h h v}\right)^{T}, \\
& \Gamma^{(3,3)}=M_{5 h} B_{h}-H_{h h v}+(*) .
\end{aligned}
$$

Finally, applying Lemma 2 gives (15), thus concluding the proof. $\square$

Remark 2: Several results can be obtained from Theorem 1, for instance, setting $\eta=0_{n \times m}$ or $\eta=B_{h}$ or $M_{5}=0_{m \times n}$. Different configurations may lead to different results [36].

Remark 3: In this particular case (SOFC design), when using the vector $\left[\begin{array}{lll}x^{T} & \dot{x}^{T} & u^{T}\end{array}\right]^{T}$, equivalent conditions are obtained employing the Finsler's Lemma and descriptor- 
redundancy. Effectively, consider $\bar{x}=\left[\begin{array}{lll}x^{T} & \dot{x}^{T} & u^{T}\end{array}\right]^{T}$; thus, the system (2) together with the control law (7) writes:

$$
\left.\begin{array}{l}
0 \times \ddot{x}=A_{h} x+B_{h} u-E_{v} \dot{x} \\
0 \times \dot{u}=H_{h h v}^{-1} K_{h v} C_{h} x-u
\end{array}\right\} \Leftrightarrow \bar{E} \dot{\bar{x}}=\bar{A}_{(\cdot)} \bar{x},
$$

with $\bar{E}=\left[\begin{array}{lll}I & 0 & 0 \\ 0 & 0 & 0 \\ 0 & 0 & 0\end{array}\right]$ and $\bar{A}_{(\cdot)}=\left[\begin{array}{ccc}0 & I & 0 \\ A_{h} & -E_{v} & B_{h} \\ H_{h h v}^{-1} K_{h v} C_{h} & 0 & -I\end{array}\right]$.

The Lyapunov function under consideration is $V(\bar{x})=\bar{x}^{T} \bar{E}^{T} \bar{P} \bar{x}$, where $\bar{E}^{T} \bar{P}=\bar{P}^{T} \bar{E} \geq 0$ and the structure on

$$
\bar{P}=\left[\begin{array}{ccc}
P_{1} & 0 & 0 \\
P_{2} & P_{3} & P_{4} \\
P_{5} & P_{6} & P_{7}
\end{array}\right] \text { with } P_{1}=P_{1}^{T}>0 \text {. }
$$

Therefore $\dot{V}(\bar{x})<0 \Leftrightarrow \bar{A}_{(\cdot)}^{T} \bar{P}+\bar{P}^{T} \bar{A}_{(\cdot)}<0$, or extending

$$
\left[\begin{array}{ccc}
\Gamma^{(1,1)} & (*) & (*) \\
\Gamma^{(2,1)} & -P_{3}^{T} E_{v}-E_{v}^{T} P_{3} & (*) \\
\Gamma^{(3,1)} & -P_{4}^{T} E_{v}+\left(P_{3}^{T} B_{h}-P_{6}\right)^{T} & \Gamma^{(3,3)}
\end{array}\right]<0
$$

with

$$
\begin{aligned}
& \Gamma^{(1,1)}=P_{2}^{T} A_{h}+P_{5}^{T} H_{h h v}^{-1} K_{h v} C_{h}+(*), \\
& \Gamma^{(2,1)}=P_{3}^{T} A_{h}+P_{6}^{T} H_{h h v}^{-1} K_{h v} C_{h}+\left(P_{1}-P_{2}^{T} E_{v}\right)^{T}, \\
& \Gamma^{(3,1)}=P_{4}^{T} A_{h}+P_{7}^{T} H_{h h v}^{-1} K_{h v} C_{h}+\left(P_{2}^{T} B_{h}-P_{5}^{T}\right)^{T}, \\
& \Gamma^{(3,3)}=P_{4}^{T} B_{h}-P_{7}^{T}+(*) .
\end{aligned}
$$

Note that considering $P_{1}=P, P_{2}^{T}=M_{1}, P_{3}^{T}=M_{3}$, $P_{4}^{T}=M_{5}, P_{5}^{T}=M_{2}, P_{6}^{T}=M_{4}$, and $P_{7}^{T}=M_{6}$ (18) gives exactly the same as (14).

\section{EXAMPLES}

In this section, a numerical example is used to illustrate the performance of Theorem 1; several configurations for the free matrix $\eta \in \mathbb{R}^{n \times m}$ have been done (see Remark 2).

Example 1. Consider a TS descriptor model of the form (2) with: $E_{1}=\left[\begin{array}{ccc}1.05 & 0.7 & 0.7 \\ -0.1 & 1.1 & -0.2 \\ 0.1 & 0.5 & 0.9-a\end{array}\right], \quad E_{2}=\left[\begin{array}{ccc}0.8+b & 0.8 & 0.7 \\ -0.9 & 1.1 & -0.2 \\ 0.4 & 0.5 & 0.6\end{array}\right]$, $A_{1}=\left[\begin{array}{ccc}-1.15 & 0.1 & 1.8+b \\ 0.3 & -1.3 & -0.5 \\ -0.1 & 0.8 & -0.8\end{array}\right], \quad A_{2}=\left[\begin{array}{ccc}-1.2 & -0.3 & -0.1 \\ 0.4 & -0.6 & 0.3 \\ -0.2 & -0.2 & -0.2-a\end{array}\right]$, $B_{1}=\left[\begin{array}{cc}0.6 & 1.2 \\ 0.3 & 1.5-a \\ -0.6 & 1.3\end{array}\right], \quad B_{2}=\left[\begin{array}{cc}-1.3 & 2.1 \\ -2.7 & 0.5 \\ 1.5 & 1.6\end{array}\right], \quad C_{1}=\left[\begin{array}{c}0.4 \\ 1 \\ 0\end{array}\right]^{T}, \quad$ and
$C_{2}=\left[\begin{array}{lll}0.8 & 1 & 0\end{array}\right]$, where $a \in\left[\begin{array}{ll}0 & 1\end{array}\right], b \in[-0.5,1]$ are realvalued parameters. Three configurations for Theorem 1 have been tested:

$$
\text { Conf }_{1}: \eta=\left[\begin{array}{cc}
0 & 0 \\
0 & 0 \\
0 & 0
\end{array}\right], \quad \operatorname{Conf}_{2}: \eta=\left[\begin{array}{ll}
1 & 1 \\
1 & 1 \\
1 & 1
\end{array}\right], \quad \operatorname{Conf}_{3}: \eta=B_{h} .
$$

Fig. 1 plots the feasible solution set for each of the configurations mentioned above: configuration 1 ( $O$ ), configuration $2(\times)$, configuration $3(\nabla)$.

Fig. 1 illustrates Remark 2, different solution sets are obtained for different selections of the matrix $\eta \in \mathbb{R}^{n \times m}$, i.e., they do not include each other.

Observe that, when configuration 3 is implemented, another sum must be taken into account, turning the problem from three sums $-\sum_{i=1}^{r} \sum_{j=1}^{r} \sum_{k=1}^{r_{e}} h_{i}(\cdot) h_{j}(\cdot) v_{k}(\cdot)$ - to four sums $-\sum_{i=1}^{r} \sum_{j=1}^{r} \sum_{l=1}^{r} \sum_{k=1}^{r_{e}} h_{i}(\cdot) h_{j}(\cdot) h_{l}(\cdot) v_{k}(\cdot)$.

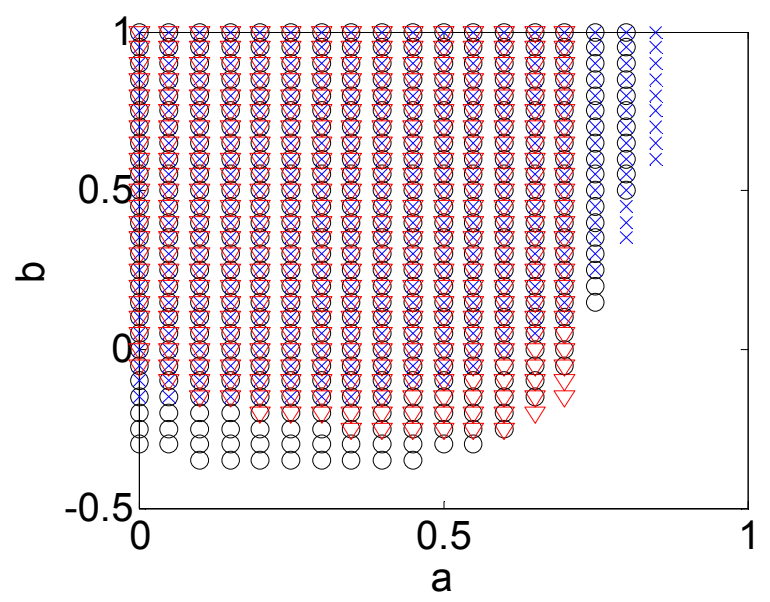

Fig. 1. Feasible solution set in Example 1.

\section{CONCLUSIONS}

An LMI approach has been presented to deal with the static output feedback controller design of nonlinear descriptor models rewritten as TS descriptor ones. Several configurations can be chosen in order to solve the problem. A numerical example has been included to show the performance of these conditions.

\section{ACKNOWLEDGMENT}

This work is supported by the Ministry of Higher Education and Research, the CNRS, the Nord-Pas-de-Calais Region (grant No. 2013_11067), a grant of the Technical University of Cluj-Napoca. The authors gratefully acknowledge the support of these institutions. 


\section{REFERENCES}

[1] T. Takagi and M. Sugeno, "Fuzzy identification of systems and its applications to modeling and control," IEEE Transactions on Systems, Man and Cybernetics, vol. 15, no. 1, pp. 116-132, 1985.

[2] K. Tanaka and M. Sugeno, "Stability analysis and design of fuzzy control systems," Fuzzy Sets and Systems, vol. 45, no. 2, pp. 135-156, 1992.

[3] S. Boyd, L. El Ghaoui, E. Feron, and V. Balakrishnan, Linear matrix inequalities in system and control theory. Philadelphia, PA: Society for Industrial and Applied Mathematics, 1994.

[4] H. Ohtake, K. Tanaka, and H. Wang, "Fuzzy modeling via sector nonlinearity concept," in 9th IFSA World Congress and 20th NAFIPS International Conference, Vancouver, Canada, 2001, pp. 127-132.

[5] K. Tanaka and H. O. Wang, Fuzzy Control Systems Design and Analysis: a Linear Matrix Inequality Approach. New York: John Wiley \& Sons, Inc., 2001.

[6] Zs. Lendek, T. M. Guerra, R. Babuška, and B. De Schutter, Stability analysis and nonlinear observer design using Takagi-Sugeno fuzzy models, vol. 262. Germany: Springer, 2010.

[7] H. O. Wang, K. Tanaka, and M. F. Griffin, "An approach to fuzzy control of nonlinear systems: Stability and design issues," IEEE Transactions on Fuzzy Systems, vol. 4, no. 1, pp. 14-23, 1996.

[8] M. Johansson, A. Rantzer, and K. E. Arzen, "Piecewise quadratic stability of fuzzy systems," IEEE Transactions on Fuzzy Systems, vol. 7, no. 6, pp. 713-722, 1999.

[9] Y. Blanco, W. Perruquetti, and P. Borne, "Stability and stabilization of nonlinear systems and Takagi-Sugeno's fuzzy models," Mathematical Problems in Engineering, vol. 7, no. 3, pp. 221-240, 2001.

[10] P. Bergsten and D. Driankov, "Observers for Takagi-Sugeno fuzzy systems," IEEE Transactions on Systems, Man and Cybernetics, Part B, vol. 32, no. 1, pp. 114-121, 2002.

[11] B. J. Rhee and S. Won, "A new fuzzy Lyapunov function approach for a Takagi-Sugeno fuzzy control system design," Fuzzy Sets and Systems, vol. 157, no. 9, pp. 1211-1228, 2006.

[12] M. Bernal, P. Hušek, and V. Kučera, "Nonquadratic stabilization of continuous-time systems in the Takagi-Sugeno form," Kybernetika, vol. 42, no. 6, pp. 665-672, 2006.

[13] T. M. Guerra, M. Bernal, K. Guelton, and S. Labiod, "Non-quadratic local stabilization for continuous-time Takagi-Sugeno models," Fuzzy Sets and Systems, vol. 201, pp. 40-54, Aug. 2012.

[14] V. C. S. Campos, F. O. Souza, L. A. B. Torres, and R. M. Palhares, "New Stability Conditions Based on Piecewise Fuzzy Lyapunov Functions and Tensor Product Transformations," IEEE Transactions on Fuzzy Systems, vol. 21, no. 4, pp. 748-760, Aug. 2013.

[15] R. Marquez, T. M. Guerra, A. Kruszewski, and M. Bernal, "Improvements on non-PDC controller design for Takagi-Sugeno models," in 2013 IEEE International Conference on Fuzzy Systems, Hyderabad, India, 2013, pp. 1-6.

[16] D. H. Lee, Y. H. Joo, and M. H. Tak, "Local stability analysis of continuous-time Takagi-Sugeno fuzzy systems: A fuzzy Lyapunov function approach," Information Sciences, vol. 257, pp. 163-175, 2014.

[17] T. González, M. Bernal, and R. Marquez, "Stability Analysis of Nonlinear Models Via Exact Piecewise Takagi-Sugeno Models," in 19th IFAC World Congress, Cape Town, South Africa, 2014, pp. 79707975.

[18] T. M. Guerra and L. Vermeiren, "LMI-based relaxed nonquadratic stabilization conditions for nonlinear systems in the Takagi-Sugeno's form," Automatica, vol. 40, no. 5, pp. 823-829, 2004.

[19] A. Kruszewski, R. Wang, and T. M. Guerra, "Nonquadratic stabilization conditions for a class of uncertain nonlinear discrete time TS fuzzy models: a new approach," IEEE Transactions on Automatic Control, vol. 53, no. 2, pp. 606-611, 2008.

[20] B. Ding, "Homogeneous polynomially nonquadratic stabilization of discrete-time Takagi-Sugeno systems via nonparallel distributed compensation law," IEEE Transactions on Fuzzy Systems, vol. 18, no. 5, pp. 994-1000, 2010.

[21] D.-H. Lee, J.-B. Park, and Y.-H. Joo, "Further theoretical justification of the k-samples variation approach for discrete-time Takagi-Sugeno fuzzy systems," IEEE Transactions on Fuzzy Systems, vol. 19, no. 3, pp. 594-597, 2011.

[22] T. M. Guerra, H. Kerkeni, J. Lauber, and L. Vermeiren, "An efficient Lyapunov function for discrete T-S models: observer design," IEEE Transactions on Fuzzy Systems, vol. 20, no. 1, pp. 187-192, 2012.

[23] Zs. Lendek, T.-M. Guerra, and J. Lauber, "Controller design for TS models using delayed nonquadratic Lyapunov functions," IEEE Transactions on Cybernetics, vol. 45 (3), pp. 453-464, 2015.

[24] D. Luenberger, "Dynamic equations in descriptor form," IEEE Transactions on Automatic Control, vol. 22, no. 3, pp. 312-321, 1977.

[25] F. L. Lewis, D. M. Dawson, and C. T. Abdallah, Robot Manipulator Control: Theory and Practice. New York: CRC Press, 2004.

[26] T. Taniguchi, K. Tanaka, K. Yamafuji, and H. O. Wang, "Fuzzy descriptor systems: stability analysis and design via LMIs," in Proceedings of the American Control Conference, California, USA, 1999, vol. 3, pp. 1827-1831.

[27] T. Taniguchi, K. Tanaka, and H. O. Wang, "Fuzzy descriptor systems and nonlinear model following control," IEEE Transactions on Fuzzy Systems, vol. 8, no. 4, pp. 442-452, 2000.

[28] V. Estrada-Manzo, Zs. Lendek, T. M. Guerra, and P. Pudlo, "Controller design for discrete-time descriptor models: a systematic LMI approach," IEEE Transactions on Fuzzy Systems, Early Access Online, DOI: 10.1109/TFUZZ.2014.2371029, 2014.

[29] V. L. Syrmos, C. T. Abdallah, P. Dorato, and K. Grigoriadis, "Static output feedback-A survey," Automatica, vol. 33, no. 2, pp. 125-137, Feb. 1997.

[30] Y.-Y. Cao, J. Lam, and Y.-X. Sun, "Static Output Feedback Stabilization: An ILMI Approach," Automatica, vol. 34, no. 12, pp. 1641-1645, Dec. 1998.

[31] C. A. R. Crusius and A. Trofino, "Sufficient LMI conditions for output feedback control problems," IEEE Transactions on Automatic Control, vol. 44, no. 5, pp. 1053-1057, May 1999.

[32] D. Huang and S. K. Nguang, "Static output feedback controller design for fuzzy systems: An ILMI approach," Information Sciences, vol. 177, no. 14, pp. 3005-3015, Jul. 2007.

[33] S.-W. Kau, H.-J. Lee, C.-M. Yang, C.-H. Lee, L. Hong, and C.-H. Fang, "Robust Ho fuzzy static output feedback control of T-S fuzzy systems with parametric uncertainties," Fuzzy Sets and Systems, vol. 158, no. 2, pp. 135-146, 2007.

[34] T. Bouarar, K. Guelton, and N. Manamanni, "Static Output Feedback controller design for Takagi-Sugeno systems - a Fuzzy Lyapunov LMI approach," in Proceedings of the 48th IEEE Conference on Decision and Control, 2009, pp. 4150-4155.

[35] H. Köroğlu and P. Falcone, "New LMI Conditions for Static Output Feedback Synthesis with Multiple Performance Objectives," presented at the 53rd IEEE Conference on Decision and Control, Los Angeles, USA, 2014, pp. 866-871.

[36] M. Chadli and T. M. Guerra, "LMI solution for robust static output feedback control of Takagi-Sugeno fuzzy models," IEEE Transactions on Fuzzy Systems, vol. 20, no. 6, pp. 1160-1165, 2012.

[37] V. Estrada-Manzo, Zs. Lendek, and T. M. Guerra, "Output feedback control for TS discrete-time nonlinear descriptor models," in 53rd IEEE Conference on Decision and Control, Los Angeles, USA, 2014, pp. 860-865.

[38] M. de Oliveira and R. Skelton, "Stability tests for constrained linear systems," Perspectives in Robust Control, vol. 268, pp. 241-257, 2001.

[39] H. D. Tuan, P. Apkarian, T. Narikiyo, and Y. Yamamoto, "Parameterized linear matrix inequality techniques in fuzzy control system design," IEEE Trans. on Fuzzy Systems, vol. 9, no. 2, pp. 324$332,2001$. 